\title{
Desempenho em confinamento e componentes do peso vivo de cordeiros mestiços de três grupos genéticos
}

\author{
Performance in feedlot and non carcass components in crossbred lambs from three \\ genetic groups
}

\begin{abstract}
Edson Luis de Azambuja Ribeiro ${ }^{\mathrm{I}^{*}}$ Humberto Cardoso Oliveira ${ }^{\mathrm{II}}$ Filipe Alexandre Boscaro de Castro ${ }^{\mathrm{II}}$ Ivone Yurika Mizubuti ${ }^{\mathrm{I}}$ Leandro das Dores Ferreira da Silva ${ }^{\mathrm{I}}$ Marco Aurélio Alves de Freitas Barbosa ${ }^{\mathrm{I}}$
\end{abstract}

\section{RESUMO}

Este trabalho teve como objetivo avaliar o desempenho em confinamento e a produção de carcaça e de componentes não carcaça de cordeiros dos grupos genéticos Texel x Hampshire Down (THD), Texel x Ile de France (TIF) e Texel x Suffolk (TS). Foram utilizados 17 cordeiros castrados, sendo seis THD, seis TIF e cinco TS, com idade de 120 dias e peso vivo médio inicial de $31,0 \pm 3,2 \mathrm{~kg}$. Os cordeiros foram confinados por um período de 51 dias, quando receberam diariamente ração concentrada comercial com $18 \%$ PB e $74 \%$ NDT, na proporção de $1,5 \%$ do peso vivo, mais cana-deaçúcar picada in natura à vontade. Não houve diferenças significativas $(P>0,05)$ para ganho de peso, peso final e escores de condição corporal entre os grupos genéticos; porém, cordeiros TS tiveram maiores $(P<0,05)$ consumos de MS do que cordeiros THD e TIF. As médias para consumo de MS, como percentagem do peso vivo, foram: 3,9; 3,6 e 3,2\%, respectivamente, para TS, THD e TIF. Não houve diferença $(P>0,05)$ entre os grupos genéticos para conversão alimentar. Pesos e rendimentos de carcaça quente também foram similares entre os grupos genéticos. Para os componentes não carcaça, houve diferenças $(P<0,05)$ para percentagem de intestino vazio e peso de coração, em que o grupo TS apresentou valores maiores. Conclui-se que, na produção de carne ovina, qualquer um dos grupos genéticos estudados pode ser utilizado, pois todos apresentaram desempenho e produção de componentes do peso vivo semelhantes.

Palavras-chave: carcaça, condição corporal, cruzamento, ganho de peso, ovinos, rendimento verdadeiro.

\section{ABSTRACT}

The objective of this research was to evaluate the performance in feedlot and the production of carcass and noncarcass components of lambs from the genetic groups Texel $x$
Hampshire Down (THD), Texel x Ile de France (TIF) and Texel $x$ Suffolk (TS). Seventeen castrated lambs were used, being six THD, six TIF and five TS. The average age and weight at the beginning of the experiment were, respectively, 120 days and $31.0 \pm 3.2 \mathrm{~kg}$. Lambs were fed lot for 51 days, where they received a daily commercial concentrate ration (18\% CP and $74 \%$ $T D N)$, in the proportion of $1.5 \%$ of body weight, and had free access to chopped in natura sugar cane. There was no significant difference $(P>0.05)$ for weight gain, final weight and body condition score among the genetic groups. However, TS lambs presented greater $(P<0.05)$ dry matter intake than THD and TIF lambs. Averages for DM intake as percentage of body weight were 3.9, 3.6 and 3.2\%, respectively, for TS, THD and TIF. There was no difference $(P>0.05)$ among genetic groups for feed conversion. Weights and dressing percentages of hot carcass were also similar among genetic groups. There were few differences among genetic groups for non-carcass components. TS lambs presented greater $(P<0.05)$ percentages of intestines and weights of heart than the other lambs. It can be concluded that in the lamb production any of the genetic groups studied can be used, for their performance and the production of live weight components were quite similar.

Key words: body condition, carcass, crossbreeding, sheep, true revenue, weight gain.

\section{INTRODUÇÃO}

O mercado consumidor de carnes está cada vez mais exigente, sendo necessário buscar alternativas para ampliar a oferta e oferecer carne com qualidade, padronização e certificação. A carne de cordeiro viabiliza a ovinocultura, impulsionando o setor a incrementar sua eficiência produtiva.

\footnotetext{
'Departamento de Zootecnia, Centro de Ciências Agrárias (CCA), Universidade Estadual de Londrina (UEL), 86051-970,

Londrina, PR, Brasil. E-mail: elar@uel.br. *Autor para correspondência.

IPrograma de Pós-graduação em Ciência Animal, UEL, Londrina, PR, Brasil.
} 
A produção de carne de cordeiro deve utilizar tecnologia adequada, aproveitando o potencial de crescimento dos ovinos jovens, utilizando-se animais com potencial para ganho de peso e uma alimentação adequada (SIQUEIRA, 1996).

O confinamento de cordeiros é estratégico no manejo da propriedade, permitindo aumentar a taxa de desfrute, a produtividade e a rentabilidade, reduzindo a pressão de pastejo durante a seca e garantindo o fornecimento de carne ovina para o mercado durante todo o ano (SIQUEIRA et al., 1993; BARROS et al., 1997). SIQUEIRA (2000) cita que o cordeiro para confinamento deve apresentar boa conversão alimentar, altos ganhos de peso e adequada deposição de gordura.

$\mathrm{O}$ uso de raças especializadas, puras ou em cruzamentos, pode melhorar sensivelmente o desempenho dos rebanhos ovinos, afetando índices produtivos e a qualidade das carcaças e carnes produzidas (KEMPSTER et al., 1987; FURUSHOGARCIA et al., 2004).

As raças tipo carne ou seus cruzamentos, geralmente, apresentam ganho de peso e características de carcaça e carne superiores às raças de duplo propósito ou raças laneiras (KEMPSTER et al., 1987; CARDELLINO, 1989; SIQUEIRA, 1990; GARCIA \& PEREIRA, 2003). As raças Ile de France, Hampshire Down, Suffolk e Texel estão entre as principais raças de corte criadas no Brasil (SIQUEIRA, 1990).

Um importante aspecto a ser considerado na produção de carne ovina são os componentes do peso vivo não pertencentes à carcaça, ou componentes não carcaça, que geralmente são desconsiderados na comercialização dos animais. De acordo com OSÓRIO (1992) e CARVALHO et al. (2007), esses componentes devem ser valorizados economicamente, pois podem ser utilizados como alimento (ex: coração, fígado e rins) ou para outras finalidades, como na indústria de vestuários (ex: pele), podendo perfazer até 60\% do peso vivo do animal. Segundo OSÓRIO et al. (1996), raça ou genótipo pode ter um efeito importante sobre a produção de componentes não carcaça.

Este trabalho teve como objetivo avaliar o desempenho em confinamento e a produção de carcaça e de componentes não carcaça de cordeiros de três grupos genéticos.

\section{MATERIAL E MÉTODOS}

O experimento foi conduzido no Sítio da Escolinha, localizado em Jandaia do Sul, Paraná (PR), com latitude $23^{\circ} 36^{\prime} 10^{\prime \prime}$ S e longitude $51^{\circ} 38^{\prime} 34^{\prime}$ ” W. O município possui temperatura ambiental média anual de $17,4^{\circ} \mathrm{C}$, precipitação média anual de $1.439,8 \mathrm{~mm}$, sendo máxima em janeiro (201,4mm em média) e mínima em junho (56,5mm em média). As análises químicas foram realizadas no Laboratório de Nutrição Animal do Departamento de Zootecnia da Universidade Estadual de Londrina, Londrina, PR. O experimento foi conduzido de agosto a outubro de 2007.

Foram utilizados 17 cordeiros castrados, com idade aproximada de 120 dias, divididos em três grupos, sendo seis mestiços Texel x Hampshire Down, seis mestiços Texel x Ile de France e cinco mestiços Texel x Suffolk. No confinamento, que foi realizado em aprisco coberto e com piso ripado e elevado do solo, os animais foram separados por grupos genéticos em baias coletivas. Os cordeiros foram vermifugados com produto à base de oxfebendazole, na dosagem de 4,5mg $\mathrm{kg}^{-1}$ de peso vivo, no início do experimento.

As rações utilizadas eram isoproteicas e isoenergéticas. Os ovinos receberam cana-de-açúcar picada (Saccharum spp L.) in natura (3,5\% PB, 1,5\% EE, 30,0\% FB, 53,0\% FDN, 35,0\% FDAe 63,0\% NDT, base seca) e ração comercial específica para ovinos (Romagnoli $^{\circledR}$, 18,0\% PB, 4,6\% EE, 19,0\% FB e 74,0\% de NDT). O concentrado foi fornecido na proporção de $1,5 \%$ do peso vivo, e a cana-de-açúcar foi fornecida à vontade, permitindo sobra de $10 \%$ da ração total. Os cordeiros tinham acesso à água e ao sal mineralizado à vontade. $\mathrm{O}$ alimento foi fornecido uma vez ao dia, às 14 horas, sendo os alimentos e as sobras pesados diariamente. O ajuste do concentrado era feito semanalmente, sempre que era feita a pesagem dos animais. As análises químicas dos alimentos e da matéria seca das sobras foram feitas segundo metodologias citadas por SILVA \& QUEIROZ (2002), e o teor de NDT foi calculado de acordo com fórmulas sugeridas por McDOWELL et al. (1974).

Os animais passaram por um período de adaptação à dieta e às condições experimentais de 20 dias. O período experimental teve duração de 51 dias. Além do peso, foram avaliadas outras características de desempenho, como o ganho de peso médio diário durante o confinamento, o escore de condição corporal no início e no final do confinamento, o consumo de matéria seca e a conversão alimentar (consumo de matéria seca/ganho médio diário).

O escore de condição corporal foi avaliado de acordo com OSÓRIO \& OSÓRIO (2005), sendo atribuídos valores de 1 a 5 , em que 1 corresponde a um animal excessivamente magro e 5, a um animal excessivamente gordo. Para avaliação, foi feita a palpação ao longo das apófises espinhosas lombares e sobre o músculo Longissimus dorsi.

Os animais foram pesados antes do abate precedido por jejum de sólidos de 16 horas. A carcaça 
foi pesada quente, sendo posteriormente armazenada em câmara fria $\left(4^{\circ} \mathrm{C}\right)$. Os componentes não carcaça (sangue, pele/lã, cabeça, patas, rúmen-retículo-omasoabomaso, intestinos, pulmão-traquéia, coração, fígado, rins, baço e gordura interna) foram pesados logo após o abate, sendo a gordura interna o somatório das gorduras omental, mesentérica, perirrenal e pélvica.

Para a obtenção do peso vivo vazio (PVV), o rúmen-retículo e os intestinos foram pesados cheios e vazios, sendo a diferença subtraída do peso vivo obtido antes do abate. $\mathrm{O}$ rendimento verdadeiro de carcaça foi determinado por meio da equação: (Peso de carcaça quente/PVV) x 100 (OSÓRIO \& OSÓRIO, 2005).

Os dados obtidos foram submetidos à análise de variância, em que a única fonte de variação foi o grupo genético. As diferenças entre médias foram comparadas pelo teste de Tukey, a nível de 5\% de probabilidade. As análises estatísticas foram realizadas no pacote estatístico SAS (1994).

\section{RESULTADOSEDISCUSSÃO}

Resultados de desempenho estão na tabela 1 , em que se observa que não houve diferença $(\mathrm{P}>0,05)$ entre os grupos genéticos. O peso médio final foi de $41,2 \mathrm{~kg}$, e o escore de condição corporal, de 4,0. Segundo OSÓRIO \& OSÓRIO (2005), esse escore de condição corporal corresponde a uma carcaça gorda; porém, sem gordura excessiva. Os autores salientaram que o estado de engorduramento ótimo de uma carcaça é aquele que corresponde à preferência do mercado consumidor.

O ganho de peso médio observado variou de 0,179 a 0,223 ( $\mathrm{P}>0,05)$, estando de acordo com o esperado. Segundo o NRC (1985), cordeiros desmamados com $30 \mathrm{~kg}$ de peso vivo e ganhos diários de $0,180 \mathrm{~kg}$ devem consumir $0,81 \mathrm{~kg}$ de NDT e $0,130 \mathrm{~kg}$ de PB. A partir do consumo de matéria seca observado (Tabela 2) e da composição estimada dos alimentos, os animais consumiram aproximadamente as quantias sugeridas pelo NRC (1985).

Os ganhos de peso observados no presente trabalho foram melhores do que os obtidos $(0,171 \mathrm{~kg})$ por CARVALHO et al. (2007) em cordeiros Texel confinados; porém, foram inferiores aos ganhos citados por CARVALHO et al. (2005) em cordeiros confinados Texel, Suffolk e Texel x Suffolk, que observaram média geral de 0,272kg. Em ambos os experimentos foram utilizados feno de Tifton-85 e concentrado na proporção de 40:60 (base seca). Além disso, os resultados foram inferiores ao ganho médio $(0,274)$ observado por PEREIRA et al. (2008) em cordeiros Santa Inês, recebendo silagem de milho e polpa cítrica úmida prensada como volumoso.

KEMPSTER et al. (1987) avaliaram o desempenho de 10 raças paternas em cruzamentos e não observaram diferenças entre cordeiros mestiços Hampshire Down, Ile de France e Suffolk para ganho médio diário de peso de carcaça. Em animais abatidos com um ano de idade e terminados em pastagem de Coastcross, RIBEIRO et al. (2000) não observaram diferenças no ganho de peso e no peso ao abate de borregos Hampshire Down e Ile de France.

Houve diferença significativa $(\mathrm{P}<0,05)$ para os consumos de matéria seca, nas diferentes formas de expressão (Tabela 2), entre os três grupos genéticos. Os animais mestiços Suffolk consumiram mais do que os mestiços Hampshire Down e mestiços Ile de France, tanto quando o consumo foi expresso em kg por animal dia, como em percentagem do peso vivo; porém, tiveram consumo similar aos mestiços Hampshire Down quando a forma de expressão foi em gramas por unidade de tamanho metabólico. Os mestiços Hampshire Down, por sua vez, consumiram mais do que os mestiços Ile

Tabela 1 - Pesos (kg) e escores de condição corporal dos cordeiros durante o confinamento.

\begin{tabular}{llll}
\hline & & & \\
Variáveis & T x HD & T x IF & T x S \\
\hline Peso inicial & $30,6 \pm 1,3$ & $31,2 \pm 1,3$ & $31,3 \pm 1,4$ \\
Peso final & $40,7 \pm 1,8$ & $40,3 \pm 1,8$ & $42,6 \pm 2,0$ \\
ECC inicial & $3,2 \pm 0,1$ & $3,1 \pm 0,1$ & $3,0 \pm 0,1$ \\
ECC final & $4,0 \pm 0,05$ & $4,0 \pm 0,05$ & $4,1 \pm 0,05$ \\
GMD $^{3}$ & $0,198 \pm 0,019$ & $0,179 \pm 0,019$ & $0,223 \pm 0,021$ \\
GECC $^{4}$ & $0,83 \pm 0,1$ & $0,92 \pm 0,1$ & $1,10 \pm 0,1$ \\
\hline
\end{tabular}

${ }^{1} \mathrm{~T}$ x HD = Texel x Hampshire Down; T x IF = Texel x Ile de France; T x S = Texel x Suffolk.

${ }^{2} \mathrm{ECC}=$ escore de condição corporal, variando de 1 = excessivamente magro a 5 = excessivamente gordo.

${ }^{3} \mathrm{GMD}=$ ganho médio diário no período total.

${ }^{4}$ GECC = ECC final - ECC inicial (ganho em escore de condição corporal no período total).

Ciência Rural, v.39, n.7, out, 2009. 
Tabela 2 - Consumo de matéria seca por animal dia, em relação à percentagem do peso vivo (PV), e por unidade de tamanho metabólico $\left(\mathrm{PV}^{0,75}\right)$ e conversão alimentar dos cordeiros durante o confinamento.

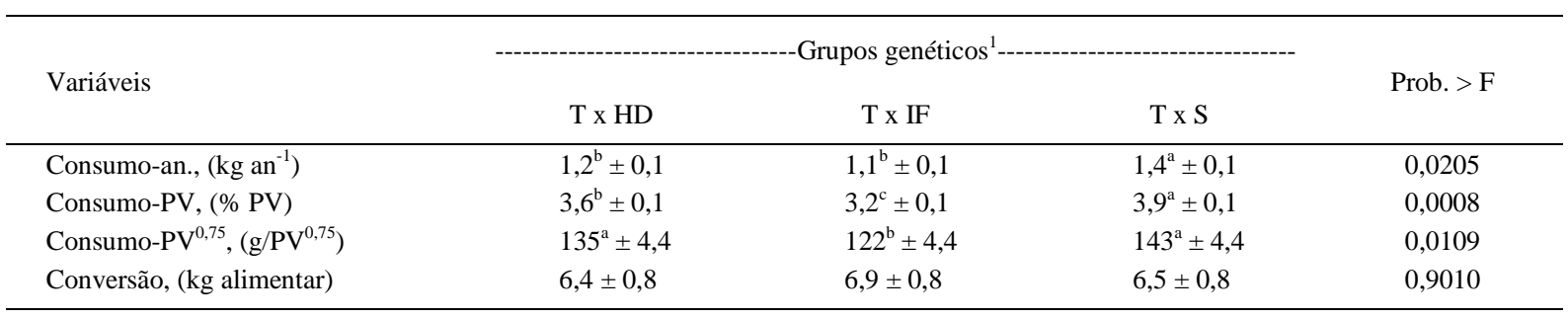

${ }^{1} \mathrm{~T}$ x HD = Texel x Hampshire Down; T x IF = Texel x Ile de France; $\mathrm{T}$ x S = Texel x Suffolk.

a,b,c - Médias na linha, seguidas de letras diferentes, diferem entre si.

de France, quando o consumo foi expresso como percentagem do peso vivo e em gramas por unidade de tamanho metabólico.

Apesar de os cordeiros mestiços Suffolk terem apresentado maior consumo, a conversão alimentar foi similar $(\mathrm{P}>0,05)$ aos cordeiros dos outros dois grupos genéticos. O valor médio de 6,6 para conversão alimentar nos indica que a dieta utilizada apresentava qualidade regular a baixa, principalmente em função da cana-de-açúcar. Usando um volumoso de melhor qualidade (feno de Tifton-85), CARVALHO et al. (2005) observaram conversão alimentar de 4,6 em cordeiros Texel x Suffolk. De maneira similar, PEREIRA et al. (2008), usando silagem de milho e polpa cítrica úmida prensada como volumosos com cordeiros Santa Inês, que teoricamente apresentam menor potencial para ganho de peso, observaram conversão alimentar de 4,3.

As médias para os diferentes consumos estão coerentes com o tipo de dieta oferecida e ganho de peso esperado. Segundo o NRC (1985), para a faixa de peso utilizada no experimento, o consumo em percentagem do peso vivo varia entre 3,5 e 4,7\%.

Os pesos de corpo vazio e de carcaça quente e os rendimentos de carcaça quente e verdadeiro foram semelhantes $(\mathrm{P}>0,05)$ entre os grupos genéticos
(Tabela 3). O rendimento de carcaça quente, apesar de estar dentro da amplitude de valores citados na literatura (SILVA SOBRINHO, 2001), pode ser considerado baixo. Uma das principais razões se deve ao alto peso do conteúdo gastrintestinal, como pode ser deduzido dos dados apresentados na tabela 4 . Apesar de os animais terem passado por um jejum de sólidos de 16 horas, eles ainda continham, no seu trato gastrintestinal, em média, 9,1kg de conteúdo, ou aproximadamente $22,1 \%$ do peso vivo final. Isso se deve, basicamente, à baixa taxa de passagem da canade-açúcar, que apresenta baixa digestibilidade da fração fibrosa (MAGALHÃES et al., 2006). CARVALHO et al. (2007) observaram 19,7\% de conteúdo gastrintestinal, após 12 horas de jejum, em cordeiros Texel confinados, em que o volumoso foi feno de Tifton-85. Os rendimentos de carcaça quente observados são próximos aos citados $(44,3 \%)$ por CARVALHO et al. (2007), para cordeiros Texel confinados, porém inferiores aos citados (49,9\%) por CARVALHO et al. (2005) para cordeiros Texel x Suffolk.

Quando os dados foram corrigidos para o conteúdo gastrintestinal, em que o peso vivo considerado para a obtenção do rendimento foi o de corpo vazio, os rendimentos foram bem superiores, com média de 56,42\%. OSÓRIO \& OSÓRIO (2005) citaram

Tabela 3 - Peso de corpo vazio (PCVazio), peso e rendimentos de carcaça quente (PCQ e RCQ) e rendimento verdadeiro (RCVerdadeiro) de cordeiros de três grupos genéticos.

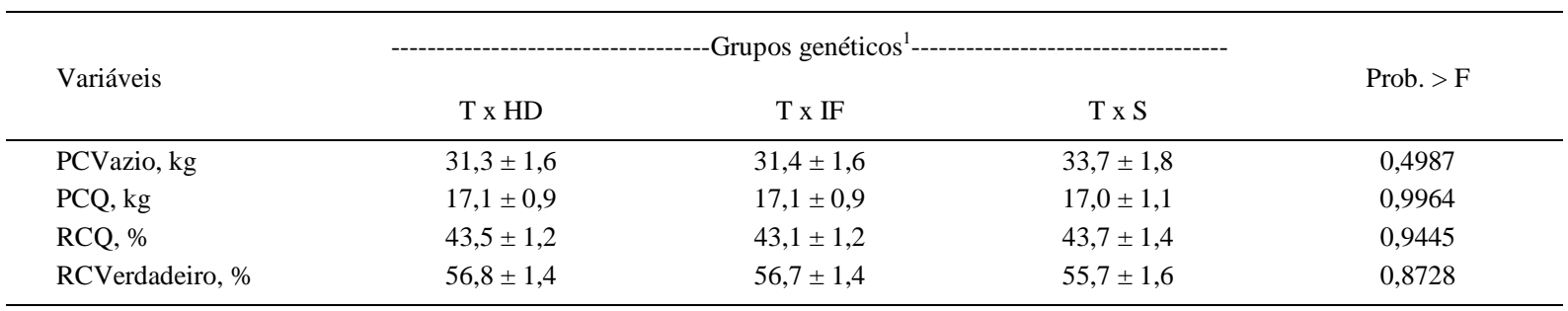

${ }^{1} \mathrm{~T}$ x HD = Texel x Hampshire Down; T x IF = Texel x Ile de France; T x S = Texel x Suffolk.

Ciência Rural, v.39, n.7, out, 2009. 
Tabela 4 - Componentes (kg) não carcaça do peso vivo de cordeiros de três grupos genéticos.

\begin{tabular}{|c|c|c|c|c|}
\hline \multirow{2}{*}{ Variáveis } & \multicolumn{3}{|c|}{-----------------------------'Grupos genéticos ${ }^{1}--------------------------------$} & \multirow{2}{*}{ Prob. $>$ F } \\
\hline & T x HD & T x IF & $\mathrm{T} \times \mathrm{S}$ & \\
\hline Pele/lã & $4,4 \pm 0,3$ & $4,3 \pm 0,3$ & $4,4 \pm 0,3$ & 0,9341 \\
\hline Cabeça & $2,1 \pm 0,1$ & $2,0 \pm 0,1$ & $2,1 \pm 0,1$ & 0,7531 \\
\hline Patas & $1,0 \pm 0,1$ & $1,2 \pm 0,1$ & $1,1 \pm 0,1$ & 0,3286 \\
\hline $\mathrm{RROA}^{2}$ cheios & $9,6 \pm 0,5$ & $8,8 \pm 0,5$ & $9,4 \pm 0,5$ & 0,5329 \\
\hline RROA vazios & $2,1 \pm 0,1$ & $1,9 \pm 0,1$ & $2,1 \pm 0,1$ & 0,3051 \\
\hline Intestinos cheios & $3,7 \pm 0,3$ & $3,7 \pm 0,3$ & $3,9 \pm 0,3$ & 0,8161 \\
\hline Intestinos vazios & $1,8^{\mathrm{b}} \pm 0,2$ & $1,7^{\mathrm{b}} \pm 0,2$ & $2,2^{\mathrm{a}} \pm 0,2$ & 0,0628 \\
\hline Pulmão-Traquéia & $0,7 \pm 0,04$ & $0,7 \pm 0,04$ & $0,7 \pm 0,05$ & 0,8397 \\
\hline Gordura interna & $0,2 \pm 0,05$ & $0,3 \pm 0,05$ & $0,4 \pm 0,05$ & 0,1143 \\
\hline Sangue & $1,4 \pm 0,06$ & $1,5 \pm 0,06$ & $1,4 \pm 0,07$ & 0,2921 \\
\hline Coração & $0,2^{\mathrm{b}} \pm 0,02$ & $0,2^{\mathrm{b}} \pm 0,02$ & $0,3^{\mathrm{a}} \pm 0,02$ & 0,0244 \\
\hline Fígado & $0,7 \pm 0,06$ & $0,8 \pm 0,06$ & $0,9 \pm 0,07$ & 0,1229 \\
\hline Baço & $0,1 \pm 0,02$ & $0,1 \pm 0,02$ & $0,1 \pm 0,02$ & 0,6466 \\
\hline Rins & $0,2 \pm 0,02$ & $0,1 \pm 0,02$ & $0,2 \pm 0,03$ & 0,1288 \\
\hline
\end{tabular}

${ }^{1} \mathrm{~T}$ x HD = Texel x Hampshire Down; T x IF = Texel x Ile de France; T x S = Texel x Suffolk.

${ }^{2} \mathrm{RROA}=$ rumen + retículo + omaso + abomaso

a,b - Médias na linha, seguidas de letras diferentes, diferem entre si.

que o rendimento de carcaça pode variar significativamente devido a vários fatores, tais como genótipo, idade, sexo e sistema de alimentação.

Nas tabelas 4 e 5, são apresentadas as médias para os componentes não carcaça em kg e percentagem do peso vivo, respectivamente. Pode-se observar que apenas a percentagem dos intestinos vazios e o peso do coração foram diferentes $(\mathrm{P}<0,05)$ entre os grupos genéticos, em que os cordeiros mestiços Suffolk apresentaram médias maiores do que os dos outros dois grupos.

Em termos percentuais, os cordeiros mestiços Suffolk apresentaram maiores médias para intestinos vazios e sangue, do que os cordeiros mestiços

Tabela 5 - Componentes (\%) não carcaça do peso vivo de cordeiros de três grupos genéticos.

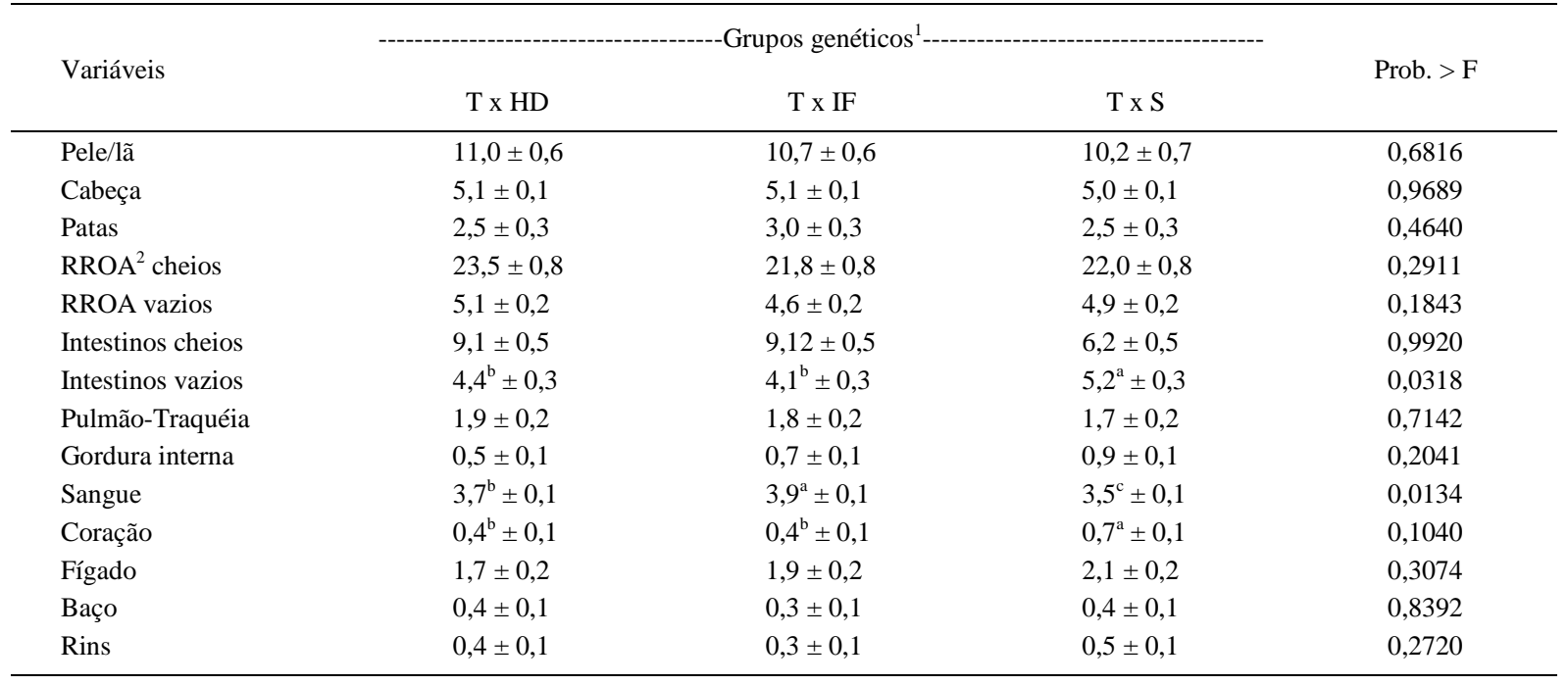

${ }^{1} \mathrm{~T}$ x HD = Texel $\mathrm{x}$ Hampshire Down; $\mathrm{T}$ x IF = Texel $\mathrm{x}$ Ile de France; $\mathrm{T}$ x S = Texel $\mathrm{x}$ Suffolk.

${ }^{2} \mathrm{RROA}=$ rumen + retículo + omaso + abomaso.

a,b,c - Médias na linha, seguidas de letras diferentes, diferem entre si.

Ciência Rural, v.39, n.7, out, 2009. 
Hampshire Down ou Ile de France. Para a maioria dos componentes não carcaça, RIBEIRO et al. (2000) também não observaram diferenças estatísticas quando compararam borregos Hampshire Down e Ile de France com um ano de idade, terminados em pastagem de Coastcross.

Em geral, as médias para as percentagens dos componentes não carcaça foram próximas das encontradas por CARVALHO et al. (2007) em cordeiros Texel terminados em diferentes sistemas de alimentação. Os autores observaram que os componentes não carcaça representaram, em média, 34,6\% do peso vivo ao abate. No presente estudo, esse valor correspondeu a $37,0 \%$.

\section{CONCLUSÃO}

Na produção de carne ovina, pode ser utilizado qualquer um dos grupos genéticos estudados (Texel x Hampshire Down, Texel x Ile de France e Texel x Suffolk), pois apresentam desempenho e produção de componentes do peso vivo semelhantes.

\section{AGRADECIMENTO}

Os autores agradecem ao Conselho Nacional de Desenvolvimento Científico e Tecnológico (CNPq) pelas bolsas de produtividade em pesquisa de Ribeiro e Mizubuti.

\section{REFERÊNCIAS}

BARROS, N.N. et al. Terminação de borregos em confinamento no nordeste do Brasil. Sobral: EMBRAPA CNPC, 1997. (Circular técnica, 12).

CARDELLINO, R.A. Sistemas de produção de carne ovina utilizando cruzamentos. In: SIMPÓSIO PAULISTA DE OVINOCUlTURA, 1989, Botucatu. Anais... Campinas: Fundação Cargill, 1989. p.97-104.

CARVALHO, S. et al. Desempenho e características da carcaça de cordeiros das raças Texel, Suffolk e cruza Texel x Suffolk. Ciência Rural, v.35, n.5, p.1155-1160, 2005. Disponível em: $<$ http://www.scielo.br/scielo.php?script=sci_arttext\&pid=S0103$84782005000500026 \& \operatorname{lng}=p t \& n r m=i s o>$. Acesso em: 06 maio 2009. doi: 10.1590/S0103-84782005000500026.

CARVALHO, S. et al. Ganho de peso, características da carcaça e componentes não-carcaça de cordeiros da raça Texel terminados em diferentes sistemas alimentares. Ciência Rural, v.37, n.3, p.821-827, 2007 . Disponível em: <http:// www.scielo.br/scielo.php?script=sci_arttext\&pid=S010384782007000300034\&lng=pt\&nrm=iso $>$. Acesso em: 06 maio 2009. doi: 10.1590/S0103-84782007000300034.

FURUSHO-GARCIA, I.F. et al. Desempenho de cordeiros Santa Inês puros e cruzas Santa Inês com Texel, Ile de France e
Bergamácia. Revista Brasileira de Zootecnia, v.33, n.6, p.1591-1603, 2004. Disponível em: <http://www.scielo.br/ scielo.php ? script = sci_arttext\&pid=S 1516 $35982004000600027 \& \operatorname{lng}=p t \& n r m=i s o>$. Acesso em: 06 maio 2009. doi: 10.1590/S1516-35982004000600027.

GARCIA, I.F.F.; PEREIRA, I.G. Manejo de cruzamentos na ovinocultura. In: SIMPÓSIO MINEIRO DE OVINOCULTURA, 3., 2003, Lavras. Anais... Lavras: UFLA, 2003. p.49-79.

KEMPSTER, A.J. et al. Growth and carcass characteristics of crossbred lambs by ten sire breeds, compared at the same estimated carcass subcutaneous fat proportion. Animal Production, v.44, p.83-98, 1987.

MAGALHÃES, A.L.R. et al. Cana-de-açúcar em substituição à silagem de milho em dietas para vacas em lactação: parâmetros digestivos e ruminais. Revista Brasileira de Zootecnia, v.35, n.2, p.591-599, 2006. Disponível em: <http:// www.scielo.br/scielo.php?script=sci_arttext\&pid=S1516$35982006000200036 \& \operatorname{lng}=p t \& n r m=i s o>$. Acesso em: 06 maio 2009. doi: 10.1590/S1516-35982006000200036.

McDOWELL, L.R. et al. Tabelas de composição de alimentos da América Latina. Gainesville: Universidade da Flórida, 1974. 63p.

NATIONAL RESEARCH COUNCIL - NRC. Nutrient requeriments of sheep. New York: National Academy, 1985. 99p.

OSÓRIO, J.C.S. Estudio de la calidad de canales comercializadas en el tipo ternasco según la procedencia: Bases para la mejora de dicha calidad en Brasil. 1992. 335f. Tese (Doutorado em Veterinária) - Curso de Doutorado em Produção Animal. Universidad de Zaragoza.

OSÓRIO, J.C. et al. Produção de carne em ovinos de cinco genótipos: componentes do peso vivo. Ciência Rural, v.26, n.3, p.471-475, 1996. Disponível em: <http://www.scielo.br/ scielo.php ? script = sci_arttext\&pid=S0103$84781996000300023 \& \operatorname{lng}=p t \& n r m=i s o>$. Acesso em: 06 maio 2009. doi: 10.1590/S0103-84781996000300023.

OSÓRIO, J.C.S.; OSÓRIO, M.T.M. Produção de carne ovina: técnicas de avaliação "in vivo" e na carcaça. Pelotas: UFPEL, 2005. 82p.

PEREIRA, M.S. et al. Consumo de nutrientes e desempenho de cordeiros em confinamento alimentados com dietas com polpa cítrica úmida prensada em substituição à silagem de milho. Revista Brasileira de Zootecnia, v.37, n.1, p.134-139, 2008. Disponível em: <http:/ /www.scielo.br/scielo.php?script=sci_arttext\&pid=S1516$35982008000100020 \& \operatorname{lng}=$ pt\&nrm=iso $>$. Acesso em: 06 maio 2009. doi: 10.1590/S1516-35982008000100020.

RIBEIRO, E.L.A. et al. Ganho de peso e componentes do peso vivo em borregos Ile de France inteiros ou castrados e Hampshire Down castrados abatidos aos doze meses de idade. Ciência Rural, v.30, n.2, p.333-336, 2000. Disponível em: <http:// www.scielo.br/scielo.php?script=sci_arttext\&pid=S0103$84782000000200022 \& \operatorname{lng}=p t \& n r m=i s o>$. Acesso em: 06 maio 2009. doi: $10.1590 / \mathrm{S} 0103-84782000000200022$. 
SAS INSTITUTE. SAS/STAT User's Guide. Cary, 1994. 1686p.

SILVA SOBRINHO, A.G. Criação de ovinos. Jaboticabal: FUNEP, 2001. 302p.

SILVA, D.J.; QUEIROZ, C. Análise de alimentos: métodos químicos e biológicos. Viçosa: Universidade Federal de Viçosa, 2002. 235p.

SIQUEIRA, E.R. Raças e sistemas de produção. In: PRODUÇÃO DE OVINOS, 1990, Jaboticabal, SP. Anais... Jaboticabal: FUNEP, 1990. p.1-25.
SIQUEIRA, E.R. et al. Estudo comparativo de recria de cordeiros em confinamento e pastagem. Veterinária e Zootecnia, v.5, p.17-28, 1993.

SIQUEIRA, E.R. Recria e terminação de cordeiros em confinamento. In: NUTRIÇÃO OVINOS, 1996, Jaboticabal, SP. Anais... Jaboticabal:FUNEP, 1996. p.175-212.

SIQUEIRA, E.R. Sistemas de confinamento de ovinos para corte no Sudeste do Brasil. In: SIMPÓSIO INTERNACIONAL SOBRE OVINOS E CAPRINOS DE CORTE, 2000, João Pessoa. Anais... João Pessoa: Empresa de Pesquisa Agropecuária da Paraíba, 2000. p.107-117. 